\title{
Light and gas confinement in hollow-core photonic crystal fibre based photonic microcells
}

\author{
F. Benabid \\ F.Benabid@bath.ac.uk \\ P. J. Roberts \\ F. Couny \\ P. S. Light
}

\author{
Centre for Photonics Photonic Materials, Physics Department, University of Bath, United Kingdom \\ DTU Fotonik, Danish Technical University, DK-2800Kgs. Lyngby, Denmark
}

Centre for Photonics Photonic Materials, Physics Department, University of Bath, United Kingdom

Centre for Photonics Photonic Materials, Physics Department, University of Bath, United Kingdom

We review the recent progress on the understanding of optical guidance mechanisms in hollow-core photonic crystal fibres, and on the quantum and nonlinear optical applications of photonic microcells based on this fibre form. Two classes of hollow-core photonic crystal fibre are identified: one guides via a photonic bandgap and the other guides by virtue of an inhibited coupling between core and cladding mode constituents. For the former fibre type, we explore how the bandgap is formed using a photonic analogue of the tight-binding model and how it is related to the anti-resonant reflection optical waveguide guidance. For the second type of fibre, which can guide over a broad wavelength range, we examine the nature of the inhibited coupling. We describe a technique for the fabrication of photonic microcells that can accommodate vacuum pressures, and we finish by showing the latest results on electromagnetically induced transparency in a rubidium filled hollow-core photonic crystal fibre, the CW-pumped hydrogen Raman laser and the generation of multi-octave spanning stimulated Raman scattering spectral combs. [D0I: 10.2971/jeos.2009.09004]

Keywords: photonic bandgap, hollow-core photonic crystal fibre, coherent effects, stimulated Raman scattering

\section{INTRODUCTION}

A photonic microcell [1] is a gas-phase photonic material whose functionality is determined by an active gas contained in a hermetic fashion within its photonic structure. Several distinct forms of photonic microcell geometry are possible, but all involve the confinement of a gas within the core of a length of hollow-core optical waveguide. The most robust form of the microcell, enabling a very high overlap between a light field and the gas, consists of a hollow-core photonic crystal fibre (HC-PCF) that is sealed at each end by low-loss splices to conventional solid optical fibres. This photonic component combines all the attributes of integrability into the existing optical network, together with a high efficiency due to the strong light and gas co-confinement. HC-PCF have been subject to an ever growing interest over the past decade, with applications spanning from telecoms [2] and metrology [1], to gas-laser systems [3]-[6]. The advent of this fibre has enabled the longstanding wish of transporting tightly focused laser beams in a gas medium without the constraints of diffraction to be realized. Furthermore, by simply filling the core, the HCPCF allows guidance in any gas-phase or liquid-phase material. As a result of these unique properties, a great effort has been devoted to the development of waveguides with a hollow core tailored to have specific properties. Figure 1 shows micrographs of a set of different hollow waveguides that have been developed during recent years. Despite their common feature of guiding light through a hollow core, these fibres neither share the same structural features nor guide via the same physical mechanism. For example, the fibres shown in Figures 1(a)-1(c) are all made with silica, and are all HC-PCF in the sense that the cladding surrounding the core forms a 2-dimensional photonic crystal. However, fibre shown in Figure 1(a), whose cladding comprises a triangular arrangement of air holes, guides via a photonic bandgap (PBG) (see Section 2). On the other hand, the cladding photonic structure of the fibre shown in Figure 1(b) constitutes a Kagome lattice, and, unlike the fibre described-above, does not exhibit a PBG. Instead, the optical guidance takes place by a mechanism akin to the formation of Von-Neumann-Wigner quasi-bound states in a continuum [7] (see Section 3). This fibre offers a loss figure of the order of a few $100 \mathrm{~dB} / \mathrm{km}$, which is much higher
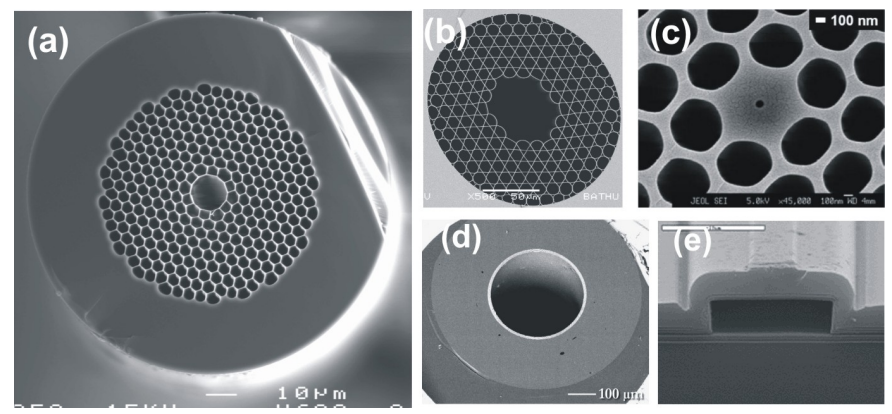

FIC. 1 Hollow-core waveguides (a) Triangular band gap HC-PCF, (b) Kagome HC-PCF, (c) Nano-void fibre, (d) Bragg fibre, (e) Chip integrated waveguide. 
than in a PBG HC-PCF. However, the Kagome HC-PCF can guide over a bandwidth which can be 20 times larger than the PBG HC-PCF. Another form of optical guidance was demonstrated with the fibre shown in Figure 1(c). The fibre has the distinctive feature of guiding with a highly enhanced intensity within a central nano-scaled hollow-core. Here, the light is confined in and close to the hollow region because of the discontinuity of the electric field at the interface between the silica surround and the hole [8].

In addition to silica-made HC-PCF mentioned-above, there are also optical hollow-core waveguides made of materials other than silica. For example, Figure 1(d) shows a hollowcore fibre made of two chalcogenide glasses and arranged in concentric layers to form what is called a Bragg optical fibre $[9,10]$. With a judicious choice of the layers' refractive indices and spacing, this fibre guides via a PBG over all the possible incident angles which correspond to propagation within the core medium (omniguidance) [11]. The last hollow-core waveguide in the list, shown in Figure 1(e), guides by virtue of an anti-resonant reflection optical wave-guidance (ARROW) mechanism. This structure has the distinctive feature of being integrated within a silicon chip [12].

In Section 2 of this paper, we introduce a simple theoretical model to explain the formation of PBG in triangular-lattice HC-PCF and demonstrate that the ARROW picture corresponds to a simple limit of this model. In Section 3, we examine the nature of the inhibited coupling between the cladding and core modes in Kagome-lattice HC-PCF. In Section 4, we describe the fabrication of photonic microcell that can accommodate all ranges of gas pressures. This section also discusses a number of recent results on electromagnetically-induced transparency in Rb-filled HC-PCF, a CW-pumped Raman gas fibre-laser, and the generation of ultra-broad comb-like coherent spectra using stimulated Raman scattering in HC-PCF.

\section{BAND GAP GUIDING HC-PCF}

\subsection{Introduction}

The fibres shown in Figures 1(a) and 1(d) guide through the trapping of the light in a core defect by an out-of plane photonic band gap (PBG), created either by the 2-dimensional photonic crystal cladding (Figure 1(a)) [13] or a set of concentric layers (Figure 1(b)) [11]. For these classes of fibre, the cladding exhibits no symmetry-compatible propagating modes at the same effective index and frequency as the guided core defect-mode. The attributes of the defect mode in this class of fibre have been extensively studied. These studies include dispersion characteristics, spatial mode field distribution, anti-crossings between core-guided and core-interface modes and the scaling laws for the PBG location (see e.g. [14][19]). Furthermore, the linear loss of such fibres could in principal reach lower levels than the present record of $1.2 \mathrm{~dB} / \mathrm{km}$ over a spectral range of $\sim 50 \mathrm{THz}$ demonstrated using the 2dimensional PBG form. The design and the theoretical studies of bandgap fibres has, until recently, relied on ab-initio numerical solutions of the Maxwell-Helmholtz equation.

$$
\left(\nabla_{\|}^{2}+k^{2} n^{2}\right) \mathbf{h}+\nabla_{\|}\left(\ln n^{2}\right) \times\left(\nabla_{\|} \times \mathbf{h}\right)=\beta^{2} \mathbf{h},
$$

where $\mathbf{h}=h_{x} \hat{\mathbf{x}}+h_{y} \hat{\mathbf{y}}$ is the transverse component of the magnetic field, $n$ represents the spatial refractive index profile of the photonic structure. $k$ is the free-space wavenumber, $\beta$ is the axial (Oz-directed) wavenumber component also called the propagation constant, and $\nabla_{\|}=(\partial / \partial x) \hat{\mathbf{x}}+(\partial / \partial y) \hat{\mathbf{y}}$ is the transverse gradient operator. When Eq. (1) is cast as an eigenvalue problem, it provides information on the existence of photonic bandgaps and their spectral location. Though powerful, this numerical method neither provides strong insight on how a PBG forms nor provides a simple predictive tool.

Recently, theoretical and experimental results identified the structural features in the cladding photonic structure which are behind the formation of the PBG in the triangular-lattice HC-PCF using an intuitive model based on the photonic analogue of tight-binding model in atomic physics [20]. The analysis showed that the PBG is formed by three types of underlying cladding mode constituents: the light at the lower frequency band-edge is confined mainly at the glass nodes which exist where three glass struts meet, whereas the upper frequency band-edge results from the interplay between a mode associated with the struts and another which is mainly localized within the holes.

In order to show how a PBG forms in microstructured optical fibres, we start by giving a general historical background, then exemplify the formation of PBG by using the photonic tightbinding model (PTBM) for a simple 1D array of optical fibres. Finally, we explain within the framework of PTBM the genesis of a PBG in a realistic triangular-lattice HC-PCF, and identify resonances of the various structural constituents of the photonic structure which are responsible for the photonic bands either side of the band gap.

\subsection{Historical background}

Among the previous work which addressed the nature of the cladding states which exist outside the band gap, and how light propagates within different constituent features of a photonic crystal cladding, there is that of Yariv and Yeh $[9,10]$. In analyzing their proposal of a Bragg fibre (i.e. a fibre with a cladding made of concentric solid annular rings with alternating refractive indices) in the 1970s $[9,10]$, the authors pointed out qualitatively that the allowed bands (i.e. cladding modes) which sandwich the bandgaps in the $\beta-\omega$ diagram relating mode propagation constant $(\beta)$ to the optical frequency $(\omega)$, are composed of constituent modes mainly confined in the high-index layers. The number of these modes is determined by the number of layers. As the frequency and the effective indices of the modes in a particular allowed band become higher, this band shrinks to a set of degenerate modes confined in the high-index layers. This observation clearly indicates the role of the resonance properties of the high-index layer in the formation of the bandgap [21]. Subsequently, Duguay et al. introduced the terminology of Anti-Resonant Reflective Optical Waveguides (ARROW) [22]. The ARROW model has since been successfully applied to some classes of photonic crystal fibres [23, 24]. These studies [23, 24] consider PCFs with a structure composed of a low-index background material with isolated high-index cylindrical inclu- 
sions. They show, using the ARROW model, that the frequencies of transmission bands are primarily determined by the geometry and the modal properties of the individual highindex inclusions irrespective of the lattice constant value. Consequently, one can consider the high-index inclusions as optical resonators whose modal properties determine the properties of the formed bandgaps. More recently, Birks et al [25] proposed a semi-analytical approach to understanding the formation of bandgaps in all-solid bandgap PCFs with the same type of cladding structure as in references [23, 24]. The authors modelled the out-of-plane mode structure of the fibre cladding by employing an approximate technique related to the cellular method used in solid-state physics [26]. This method explicitly takes into account electromagnetic coupling between each high index cylinder within the cladding and, in contrast to the ARROW model, provides information on the width of the cladding pass-bands. The model also elucidates the hybridization which occurs between resonances associated with the localized high index regions and the low index regions which fit between them.

The "cellular method" proposed in [25] provides important and useful insights into the guidance mechanism of all-solid PBG photonic crystal fibres which comprise arrangements of separated high index regions within a lower index background, and on the nature of bandgap formation within the claddings of these fibres. However, the complex topological structure of the silica-air PCF cladding, such as possessed by the HC-PCF shown in Figure 1(a), makes it very challenging to identify the relevant optical resonators responsible for the PBG formation. Indeed, the singly-connected nature of the high index component of these fibres calls into question the applicability of a picture of guidance and band gap formation based on the influence of a few constituent elementary resonator features. Consequently, in order to assess the applicability of a resonator picture as an aid to designing realistic HC-PCF, it is useful to develop experimental or theoretical tools aimed at identifying any relevant cladding features which may be present.

\subsection{Photonic tight-binding model: Toy model for PBG formation}

The tight binding model has been successfully used in atomic and solid-state physics to give an approximate description of the origin of electronic bands (allowed and forbidden) [26]. In the tight-binding picture, electronic bands in a solid (e.g. a crystal) are considered to be the result of "bringing" together isolated and identical atomic sites which constitute the solid. This approach has the double advantage of holding a high degree of predictive power as well as being simple. The model starts with the Hamiltonian of a single atom with its well defined energy states and wavefunctions. As these sites get closer, the set of degenerate single electronic states, each of which has a wavefunction (orbital) well localized by the potential well created by an atom, spread to form a band of energy levels associated with delocalized wavefunctions (Bloch functions). The wavefunctions are all a linear superposition of the isolated atomic orbitals. Furthermore, the upper energy level of the formed band is associated with a wavefunction which changes sign between nearest-neighbours atomic sites, and the lower energy level is formed by a symmetric tightly-bound state with no phase oscillations. We expect that a simple transposition of the tight-binding model to a photonic structure will provide a wealth of information on the nature of the modes in the allowed bands and the formation of bandgaps.

Let us now consider a one-dimensional array of $N$ identical optical fibres. Each fibre consists of a silica rod with radius $\rho$, and the fibres are spaced by a pitch $\Lambda$. Figure 2(a) shows the dispersion curves for the fundamental mode and the degenerate second order modes. For simplicity, the curves were deduced from an approximate analytical expression of the propagation constant for the fundamental and the second-order modes of the individual fibres [27].

$$
\beta^{2}=\left(n_{c o} k\right)^{2}-\frac{U_{\infty}^{2}}{\rho^{2}} \frac{k^{2}}{(k+\eta / \rho)^{2}}
$$

Here, $U_{\infty}$ is a constant equal to 2.405 and 3.832 for the fundamental mode and the second order modes, respectively. $\eta$ is a function of the index of the rod, $n_{c o}$, and that of the background, $n_{b k g}$, given by $\eta=(1-\Delta) / n_{c o} \sqrt{2 \Delta}$, with $\Delta=$ $\left(n_{c o}^{2}-n_{b k g}^{2}\right) / 2 n_{c o}^{2}$. In our example, the $n_{c o}$ is taken to be 1.45 and $n_{b k g}=1$ (i.e. air). The case of an array of $N$ silica rods can be solved using an approach based on coupled mode theory, which can be considered equivalent to a tight binding model. For the sake of simplicity, we only account for the coupling due to adjacent rods. Furthermore, we consider a Gaussian profile for the rod index so that the coupling coefficient, $C$, between two identical rods is given by the following simple analytical expression which is valid for the fundamental modes and the second order modes [28]

$$
\begin{aligned}
C \Lambda= & \left(\sqrt{2 \pi} n_{c o} \Delta \tilde{\rho}\right) k \Lambda \sqrt{n_{c o} \sqrt{2 \Delta} k \Lambda} \\
& \times \exp \left[-(\tilde{\rho}-1)\left(\tilde{\rho}^{-1}-n_{c o} \sqrt{2 \Delta} k \Lambda\right)\right]
\end{aligned}
$$

Here $\tilde{\rho}=\rho / \Lambda$ is the normalized radius.

The set of the coupled equations is reduced to an eigenvalue problem involving the following tri-diagonal $\mathrm{N}$-dimensional matrix

$$
\left(\begin{array}{cccccc}
\beta & C & 0 & 0 & \ldots & 0 \\
C & \beta & C & 0 & \ldots & 0 \\
0 & C & \beta & C & \ldots & 0 \\
\ldots & \ldots & \ldots & \ldots & \ldots & \ldots \\
0 & 0 & 0 & 0 & C & \beta
\end{array}\right)
$$

Consequently the individual modes of the isolated rods form $N$ closely spaced modes (a continuum for the case of $N \rightarrow \infty$ ), whose propagation constant is given by

$$
\beta_{j} \Lambda=\beta \Lambda-2 \sqrt{(C \Lambda)^{2}} \cos \left(\frac{j \pi}{N+1}\right), \quad j=1,2, \ldots N
$$

Figure 2(b) shows the dispersion of these modes for $N=1000$ and $\rho=0.45 \Lambda$. The plot clearly shows the formation of the allowed photonic bands whose index-spread increases as the normalized frequency gets smaller. Furthermore, the plot also illustrates the existence of a PBG which extends even for effective indices lower than 1, thus indicating a possibility of trapping light in a hollow defect. In order to draw an analogy with 
(a)

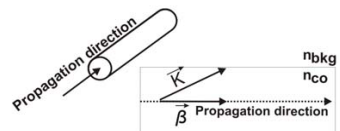

SILICA OPTICAL-FIBER ROD

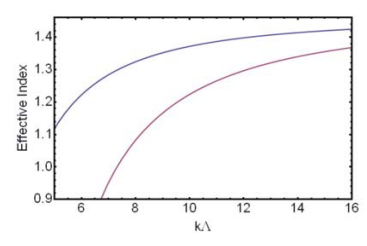

DISPERSION CURVES OF A SILICA ROD

(c)

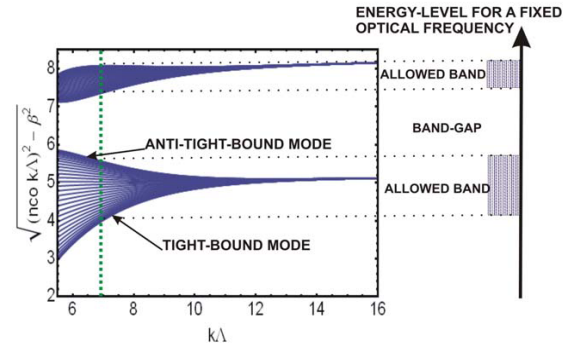

FIG. 2 Photonic Tight-Binding model: (a) Dispersion curve of fundamental and second higher-order modes of a silica rod. (b) Dispersion diagram of an array of silica rods. separated by a pitch $\Lambda$. (c) Re-representation of the dispersion diagram with ordinate $\sqrt{\left(n_{c o} k\right)^{2}-\beta^{2}}$, revealing more clearly the nature of the photonic bandgaps.

the tight-binding model in atomic physics, Figure 2(c) shows the same dispersion diagram as Figure 2(b), but the effective index is replaced by $\left[\left(n_{c o} k\right)^{2}-\beta^{2}\right]^{1 / 2}$. This figure shows the splitting of the fundamental mode and its transformation into an allowed band as $k \Lambda$ decreases, which can be achieved by moving the rods closer to each other at a fixed frequency. Furthermore, by direct analogy with the tight-binding model, the mode of the lowest energy corresponds to the most strongly confined mode (tight-bound mode). This mode is also named the fundamental space-filling mode in the community of PCF [29]. Finally, one can see that the PBG becomes weakly dependant on $k \Lambda$ as $k \Lambda$ becomes large, and that the band-edges are solely determined by dispersion curves of single rod modes. This trend is also a characteristic of the ARROW model [23]. Consequently, the PTBM offers a simple and powerful framework for the study of the formation of PBG in photonic structures, as well as unifying the ARROW model with that of PBG formation.

\subsection{Photonic tight-binding model description of HC-PCF cladding}

The previous section illustrated a toy model of how a PBG forms using an approach analogous to the tight-binding model. Here, we report on experimental and theoretical results which extend this approach to describe a realistic HC-PCF (Figure 3(a)). Figures 3(d)-3(g) show the numerically calculated density-of-states (DOS) [30, 31] and the modal properties of a typical HC-PCF cladding structure (Figure 3(b)). Either side of the out-of-plane PBG region (shown in black in Figure 1(d)) are allowed bands where the cladding can support propagating modes (regions 1 and 2 in Figure 3(d)). These bands follow the PTBM in the sense that the bands shrink as $k \Lambda$ increases. Of particular
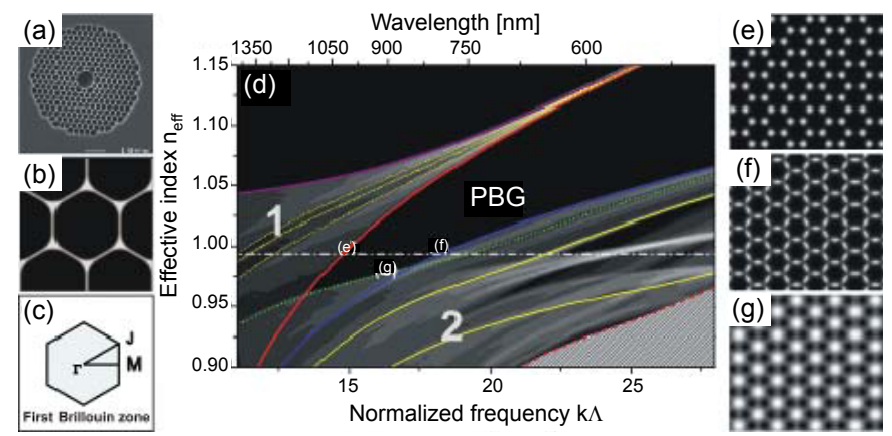

FIG. 3 (a) SEM of a HC-PCF with core guidance at $1064 \mathrm{~nm}$. (b) Details of the cladding structure used for the numerical modelling. (c) Brillouin zone symmetry point nomenclature. (d) Propagation diagram for the HC-PCF cladding lattice. Black represents zero DOS and white maximum DOS. The upper $x$-axis shows the corresponding wavelengths for a HC-PCF guiding at $800 \mathrm{~nm}$ (pitch $\Lambda=2.15 \mu \mathrm{m}$ ). The solid and dotted lines represent the $\Gamma$-point and J-point mode trajectories, respectively. The trajectory of the cladding modes on the edges of the PBC are represented in red for the interstitial "apex" mode with the near field (e), in blue for the silica "strut" mode ( $f$ ) and in green for the "air-hole" mode (g). The first two modes are shown at an effective index of 0.995 (dash-dotted white line), whereas the air-hole mode is shown at $k \Lambda=15.5$ and $n_{\text {eff }}=0.973$.

interest is the mode forming the lower-index edge of region 1 since it determines the lower-frequency edge of the PBG of the cladding at the air light-line $\left(n_{e f f}=1\right)$. The dispersion of this mode is represented in red in Figure 3(d) and its near-field (NF) is shown in Figure 3(e) at a representative normalized wavenumber $k \Lambda$. This confirms that the light is predominantly guided in the interstitial apexes. The apexes are thus identified as the most important optical resonators associated with the upper bandgap edge [18]. The nature of the modes at the upper-frequency edge of the PBG is more complicated than those at the lower-frequency edge. Indeed, the lower edge is formed by several and overlapped allowed photonic bands resulting in it being formed by a combination of the trajectories of two cladding modes of different symmetry. At frequencies (i.e. $k \Lambda$ ) below $k \Lambda=16.9$, this edge is due to a mode located at the J-point of the Brillouin zone (Figure 1(c), dotted green curve in Figure 3(d)) which guides predominantly in the air-holes ("air-hole" mode). Above $k \Lambda=16.9$, the edge is due to a mode located at the $\Gamma$-point (Figure 1(f), blue traces in Figure 1(d)) primarily guiding within and close to the silica struts ("strut" mode) which join neighboring apexes, with little light penetrating into the apexes. Consequently, the silica struts directly limit the upper-frequency of the fibre transmission band.

These observations were corroborated experimentally by measuring spectrally-resolved near field (NF) images and spatially resolved transmission spectra of $3 \mathrm{~mm}$ long $\mathrm{HC}$ PCFs using a scanning near-field optical microscope (SNOM). Both the NF and spectra were taken by exciting the fibre with super-continuum light over a narrow angular range near the air light-line. The transmission spectra of the relevant modes show distinctive cut-offs relating to the fibre PBG location. Figures $4(\mathrm{a})-4$ (c) show the typical measured NF profiles when the fibre is excited by light near the lower-frequency bandgap edge and the upper-frequency edge Figures 4(a) and 4(b) and $4(\mathrm{c})$, respectively). The NF is obtained by scanning the 
SNOM tip, with $0.15 \mu \mathrm{m}$ step, over an area covering a few unit cells of the HC-PCF cladding structure. Figure 4(a) clearly shows that the imaged mode corresponds to that of the apex resonator whilst Figures 4(b)and 4(c) shows light confined predominantly in the struts and air, respectively. Moreover, when the tip is aligned with an interstitial apex, the transmission spectra of two different fibres show cut-offs around the lower frequency edge of the PBG (see the solid black line on the lower graph of Figure 4(e) for the $1060 \mathrm{~nm}$ HC-PCF). This corresponds to the apex mode frequency cut-off near the air-line in accordance with the numerical simulation (see Figure 3(d)). Similarly, for strut and air-hole modes, the transmission spectra show a clear cut-off at the short wavelength side of the HC-PCF transmission bandwidth (see the grey line of Figure 4(d) for the $800 \mathrm{~nm}$ HC-PCF and the grey dotted line of the lower graph of Figure 4(e) for the $1060 \mathrm{~nm}$ HC-PCF). However, due to the limited spatial resolution of the SNOM and to the hybridization between the two constituent resonators, the transmission spectra collected when the tip was aligned on top of a strut or in an air hole do not show a measurable difference in their frequency cut-off. Nevertheless, the above results using the SNOM do confirm that the PBG is formed by the interplay of three distinct resonators.
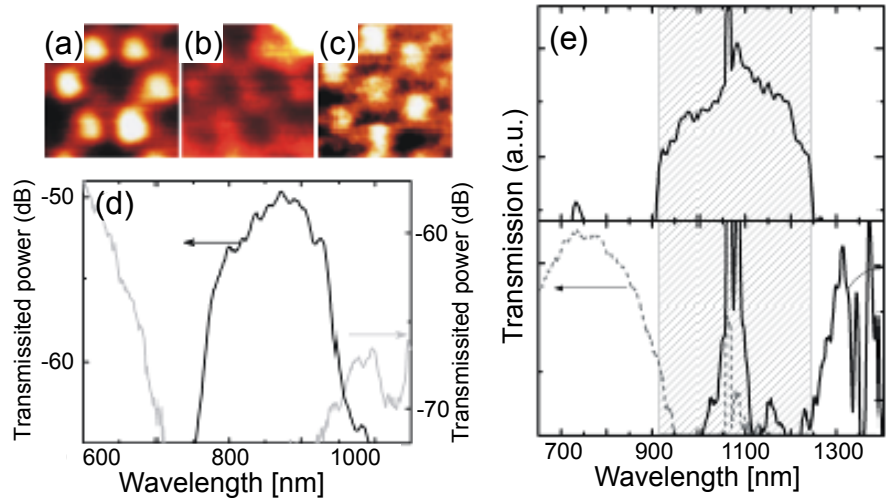

FIC. 4 SNOM images of the (a) "apex" mode (b) "strut" mode and (c) "air-hole" mode of the fibre cladding. (d) Optical spectrum of the HC-PCF guiding around $800 \mathrm{~nm}$ taken with the SNOM tip aligned with the core (black line) and near an air-hole of the cladding (grey line), (e) Optical spectrum of the HC-PCF guiding around $1064 \mathrm{~nm}$ taken with the SNOM tip aligned (top) with the core, (bottom) with an interstitial apex (black solid line) and with an air hole of the cladding (grey doted line). The peaks around $1064 \mathrm{~nm}$ are due to the residual super-continuum pump.

\section{BROADBAND GUIDING HC-PCF}

\subsection{Introduction}

The possibility that a bound or a quasi-bound state could exist within a continuum was suggested in a remarkable theoretical proposal by Von Neumann and Wigner (VNW) back in the early days of quantum mechanics [32]. However, the experimental confirmation of the existence of such states was hard to achieve because of the unusual geometry of the potentials involved. It was only in 1992, when Capasso et al [33] reported on the observation of an electronic bound state above the potential barrier in a thin semiconductor layered structure, that VNW electronic bound states were experimentally observed. The rapid development of photonic crystals dur- ing the last decade, and the conceptual similarity between photonic bandgap physics and solid-state electronic bandgap physics [34, 35], raise the question of whether the photonic analogue of bound states within a continuum could exist, and if so, whether they could be observed.

Here we report on an experimental and theoretical account of the optical guidance of a quasi-localized guided mode (i.e. photonic quasi-bound state) which has a frequency and effective index that lies within a continuum of propagating modes of the same photonic structure. The first structure considered is a HC-PCF based on a Kagome lattice cladding. We attribute the guiding within a continuum to the inhibited interaction between the transversely fast-oscillating modes associated with the silica within the cladding photonic structure and the slowly-varying air core modes.

Guidance within the core of a conventional HC-PCF, with a triangular arrangement of air-holes, relies on the existence of a full photonic bandgap (PBG) over appropriate optical frequency and effective index ranges $\left(k \Lambda, n_{\text {eff }}\right)$. Figure 5(a) summarises the linear properties of this form of fibre. The density of photonic states (DOPS), plotted as a function of the optical normalized frequency $k \Lambda$ and effective index $n_{\text {eff }}$, calculated for the cladding structure sketched on the top-right corner of Figure 5(a)), shows a region where the DOPS falls to zero (i.e. PBG). In analogy with a quantum mechanical description, the defect state (guided, bound) lies within the bandgap region (see the "energy" level diagram in Figure 5). In contrast, the Kagome-lattice HC-PCF (Figure 5(b)) shows weakly guided core-modes (of order $1 \mathrm{~dB} / \mathrm{m}$ confinement loss) even at $\left(k \Lambda, n_{\text {eff }}\right)$ where the photonic structure of the cladding exhibit a continuum of photonic states. Indeed, unlike the PBG HC-PCF, the band diagram of the Kagome lattice does not exhibit any $\left(k \Lambda, n_{e f f}\right)$ ranges near the air light-line where the DOPS falls to zero. Nevertheless, the numerical cal-
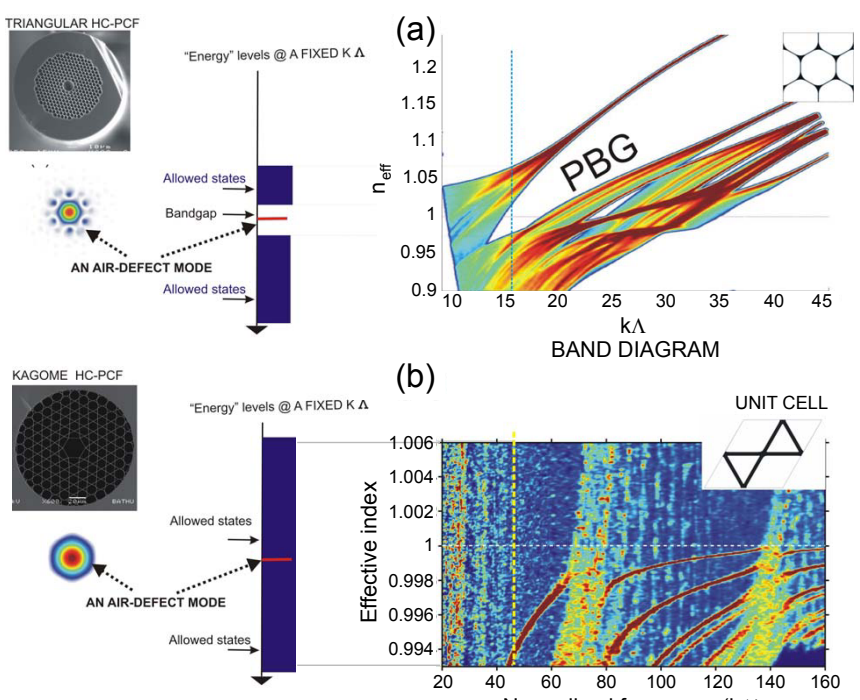

(b)

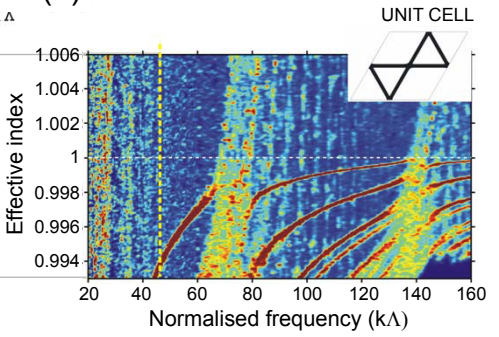

FIG. 5 (a) Top Left: Scanning electron micrograph of a triangular-lattice HC-PCF, Bottom left: near-field profile of the fundamental air-guided core mode lying within a bandgap (Center). Right: Band diagram showing the presence of the PBG (b) Same as (a) for Kagome-lattice HC-PCF. The fundamental mode lies within a continuum of cladding modes and the band diagram doesn't exhibit a PBG. 


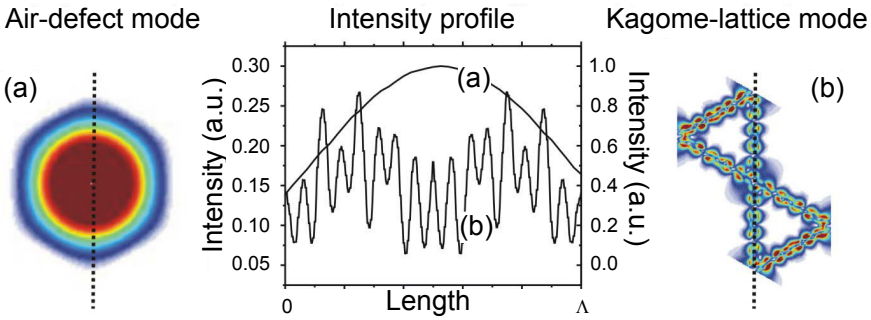

FIG. 6 Nature of the Kagome HC-PCF modes. (a) A low-loss core guided mode. (b) The mode field intensity of the cladding mode over a unit cell. (center) The intensity variation of the core mode along the dotted line in (a) and of a cladding mode along a cladding strut in (b).

culations and the experimental results show a relatively lowloss guided core mode [7].

This weak interaction between highly longitudinally phasematched core-mode and cladding modes of the same symmetry class is explained by the strong transverse-field mismatch between the modes, as evidenced by the fast intensity oscillations of the cladding mode (see Figure 6(b)), leading to the "washing-out" of the overlap with the slowly varying corefield distribution. A weak intensity overlap between core and cladding modes of a Kagome fibre was noted in [36], but we emphasize here that the inhibited interaction between core and cladding constituents is primarily due to the phase oscillations shown by the latter away from resonances which occur near $k \Lambda=\pi j(\Lambda / t) /\left[n_{g}^{2}-n_{e f f}^{2}\right]^{1 / 2},(j=1,2, \ldots .$.$) , with t$ the glass thickness and $n_{g}$ the refractive index of glass. The incompatibility between core and cladding modes can be further explored by first considering a structure which comprises concentric hexagonal layers of high index material separated by air gaps [33]. This structure behaves similarly to a true Bragg fibre with cylindrical symmetry in which modes decouple according to their azimuthal variation. The introduction of connecting struts can be considered within a perturbation theory framework if a scalar field approximation is adopted. Such a calculation leads to overlap integrals between core and cladding field constituents which become dramatically reduced by the oscillatory nature of the latter.

Figure 7 shows closely phase matched modes of a Bragglike fibre which includes connecting struts. One could anticipate an improved transmission in this class of fibre by optimizing the shape and reducing the number of connecting struts. It is worth noting that the co-existence of states with the same symmetry superficially might appear to violate the "non crossing rule". In fact, as was pointed out by Von Neumann and Wigner [32], this rule ceases to hold where dense continua are involved, so co-existence is possible even in the presence of weak residual interaction. This type of guiding and trapping of discrete photonic modes could be extended to any photonic structure. From the fabrication point of view, such structures should be less challenging than bandgap forms since they involve lattice pitches much larger than the operating wavelengths.

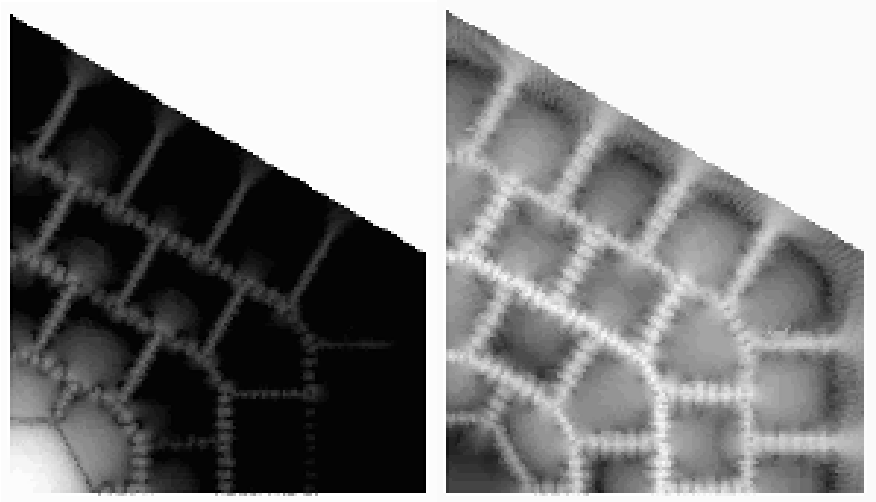

FIG. 7 Quasi-non interaction between the core mode and one of the several cladding modes which is nearly phase matched to it, in a Bragg-like HC-PCF. The mode intensity profiles are shown on a log scale over a $60 \mathrm{~dB}$ range.

\section{PHOTONIC MICROCELLS AND HC-PCF GAS-CELL APPLICATIONS}

The understanding of the physical processes behind hollow core guidance identified above allow for the optimization of the fibre's optical properties, as well as the customization of the fibre to a particular application. The enhanced gaslaser interaction that can be achieved when the fibre's core is filled with an active gas has shown its potential through the low power threshold experimental observation of nonlinear and quantum optics effects that would otherwise require GigaWatt peak-power pump lasers. Furthermore, coherent effects in molecular gases such acetylene have been demonstrated for the first time using $\mathrm{CW}$ coupling lasers. These include electromagnetically-induced transparency $[4,6]$ and saturable absorption $[37,38]$.

These demonstrations have been achieved in a compact and integrable system by the realization of a photonic microcell (PMC) [1]. The cell consists of a length of gas-filled HC-PCF that is hermitically sealed to solid optical fibres with a minimum of splice loss. The following sections highlight recent developments, both in the assembly of the photonic microcells and in their applications.

\subsection{Gas loading and microcell assembly}

The loading of high-pressure gas inside HC-PCF is achieved by first purging the fibre with $\mathrm{He}$ or Ar and then loading the active gas by applying differential pressure between the two fibre ends. The fibre can then be spliced to conventional fibre without contamination from the atmosphere so as to obtain a compact photonic microcell (Figure 8).

The realization of a microcell at vacuum pressure is more demanding. Indeed, during the splicing process, the fibre spends a limited amount of time with one end open to the atmosphere, and as a consequence, the risk of contamination of the low-pressure gas by atmospheric gases is present. In order to lift this limitation, a new technique has been developed where the fibre is prepared at the required vacuum pressure (Step 1 of Figure 8) and loaded with He at slightly more than 1 bar before being spliced (Step 2). The pressure of He is such 

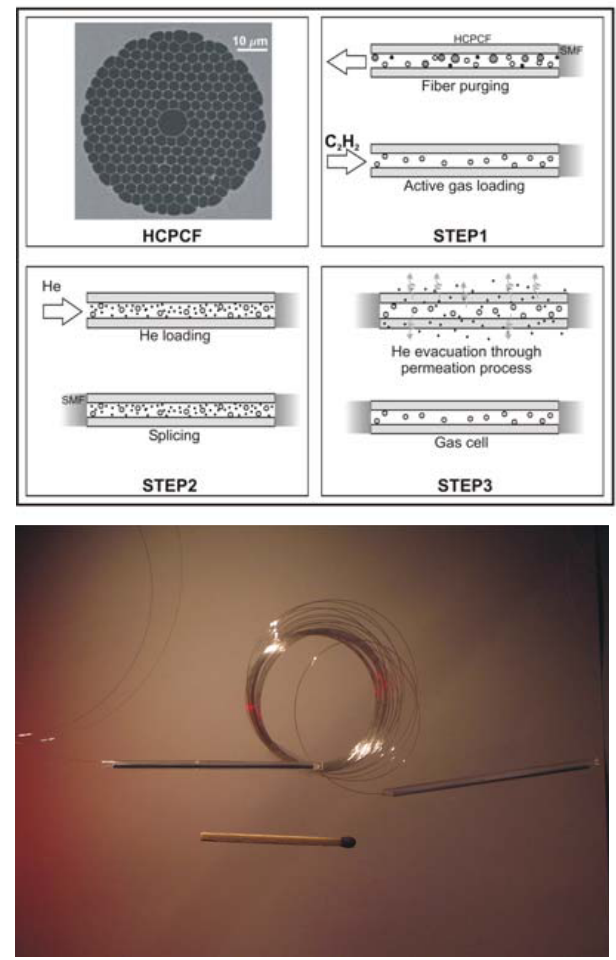

FIG. 8 Top: Photonic microcell preparation process. The various steps are described in the text. Bottom: A photonic microcell is compared in size to a match stick.

that very little contamination is possible during the splicing process. The high permeability of the He atoms through glass means that the initial vacuum pressure will be restored after a few hours, leaving a low insertion loss, compact, $\mu$ bar pressure microcell.

\subsection{Rubidium loaded HCPCF}

Among the first successful demonstrations involving HC-PCF in quantum optics and laser metrology are laser stabilization [1] and EIT [4, 6], using acetylene molecules confined in the core of these fibres. These studies have shown that strong signal-to-noise ratios and huge nonlinearities can be obtained, despite the small dipole strengths involved. Consequently, the combination of alkali metals (e.g. Rb, Cs, etc) and HC-PCF holds the promise of observing exciting coherent phenomena with just a single photon. However, the confined geometry of HC-PCF and extreme physiochemical reactivity of the alkali metal vapors renders the loading process challenging. On the other hand, the strong coherence dephasing due to collisions with the core-wall initially put a doubt on the reliability of HC-PCF for coherent optics applications. Fortunately, recent results have shown that coating the fibre core with a polymer layer could act as an atomic vapor source via light induced atom desorption (LIAD) [39] and reduce the relaxing effect of collisions with the wall [38]. Figure 9 summarizes the results of EIT generation in Rb-filled HC-PCF with a transparency linewidth much lower than that set by collisions with the uncoated silica wall. The photonic microcell form of atomic vapors filled would open new prospects in both fundamental research in quantum optics and telecommunication and optical processing. (a)

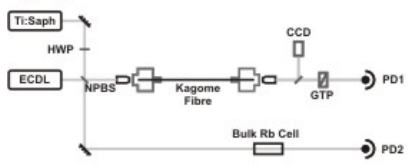

(b)

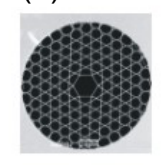

(c)

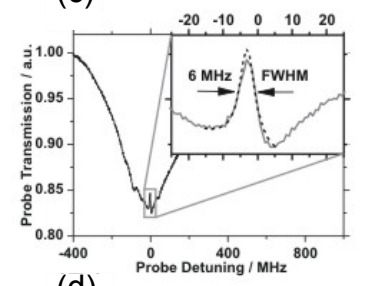

(d)

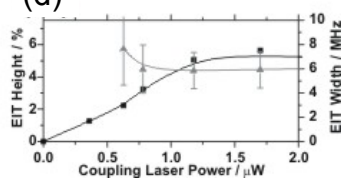

FIG. 9 EIT in Rb-filled kagome HC-PCF with PDMS coated core. (a) Schematic experimental setup. (b) Electron micrograph of the fibre cross-section, and diagram on the rubidium energy levels forming the $D_{2}$ line. (c) EIT spectrum with narrow $6 \mathrm{MHz}$ linewidth. (d) Evolution of the induced transparency height and linewidth with coupling laser power.

\subsection{First CW Raman gas fibre-laser}

Early results obtained for the generation of stimulated Raman scattering (SRS) in $\mathrm{H}_{2}$-filled HC-PCF indicated that Raman conversion in the $\mathrm{CW}$ regime could be possible with a pump power of only a few Watts. In such a single pass configuration, there are no restrictions on the converted wavelength and no requirement for a cumbersome cavity-locking system. As shown in Figure 10, the conversion is also extremely efficient with $99.99 \%$ of the output power being at the converted Stokes wavelength. These unprecedented results could widen the wavelength range achievable with $\mathrm{CW}$ laser, without compromising on the linewidth or laser power.

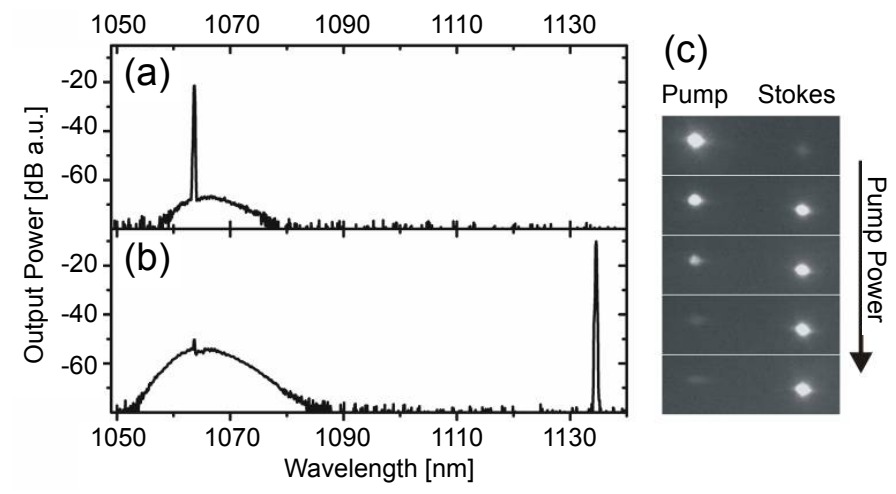

FIC. 10 CW Raman gas fibre-laser. Left: Transmission spectrum (a) below and (b) above threshold. (c) Evolution of the output intensity profile with pump power, at pump and Stokes wavelengths.

\subsection{Multi-octave spanning frequency comb generation}

An astonishing consequence of the high interaction efficiency obtained using HC-PCF is the fact that the transient regime of Raman amplification, which was previously limited to femto-second pump pulses, can be achieved with nanosecond pulses. This spawned interest from the ultra-fast optics community since the fibre could potentially generate broad coherent frequency combs, by cascaded transient Raman scattering, in the nanosecond regime. These coherent combs could 
then be recombined to create attosecond pulse trains. Thanks to the recently identified Kagome fibre guidance, frequency combs of up to 45 spectral components, covering $1000 \mathrm{THz}$, have been generated and guided through the fibre with little loss (Figure 11). This solution presents a low-power alternative to other ultra-short pulse generation methods and does not pose any engineering challenges such as cryogenic cooling.
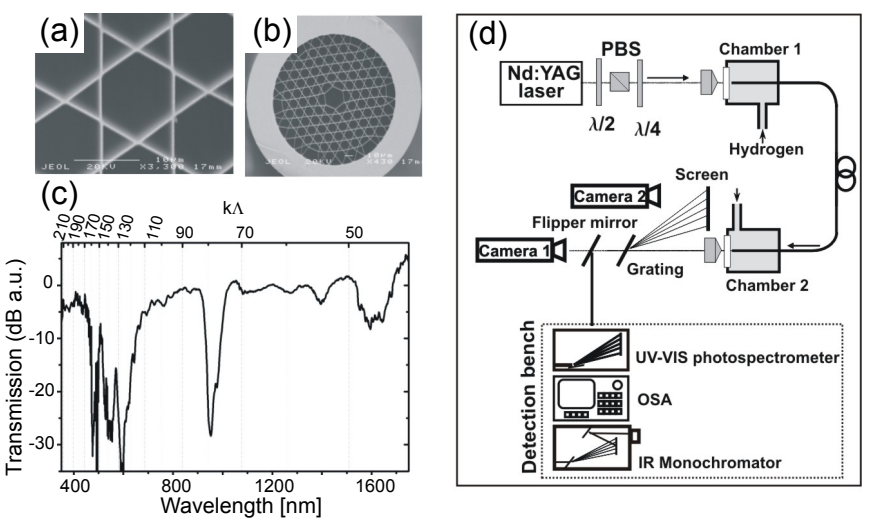

FIG. 11 (a) SEM showing the cladding structure of the Kagomé HC-PCF. (b) Lower magnification SEM showing the full structure of the fiber. (c) Optical spectrum showing the broadband guidance of the fiber, (d) Experimental setup for the generation of multi-octave frequency comb.
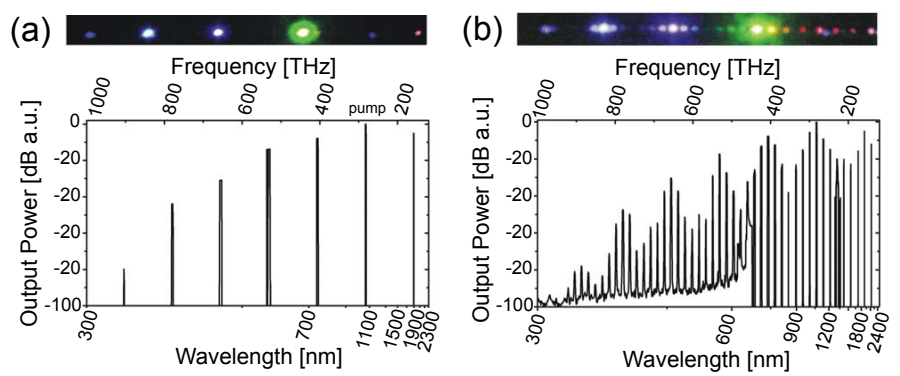

FIG. 12 Images and spectrum of the generated HSRS at the output of $\sim 1 \mathrm{~m}$ long hydrogen filled Kagomé fiber, for (a) a linearly polarized and (b) a circularly polarized laser input.

\section{CONCLUSION}

The versatility of HC-PCF has already enabled its use in applications spanning from slow light to attoscience. The ability to accurately predict the guiding properties of a particular fibre means that it can be optimized for a particular application. Simple conceptual frameworks for understanding the guidance mechanisms are an invaluable complement to $a b$ intio numerical solutions of Maxwell's equations, and serve to greatly speed up the design process. Central to the understanding of the guidance of both band-gap and Kagome fibre forms is the identification of constituent resonator components, and an appreciation of the interaction which exists between them.

The co-confinement of diffraction-free light and a gas within a cell formed using HC-PCF enables unprecedented low power levels for the observation of nonlinear and quantum optical phenomena: the waveguide makes possible light-gas interaction over several meters of propagation within a core with a size of order $100 \mu \mathrm{m}^{2}$ or less. Demonstrations of electromagnetically induced transparency in acetylene and $\mathrm{Rb}$ have shown promise for laser frequency stabilization and frequency metrology. Saturable absorption has also been observed in acetylene-filled HC-PCF, showing a line width far below the limit imposed by Doppler broadening on noncooperative processes which involve interaction with a gas. The enhanced Raman frequency generation efficiency has enabled a CW pumped Raman laser to be realized with $99.99 \%$ of the output power being at the converted Stokes wavelength in a single-pass configuration. Exploiting the broadband guiding properties of the Kagome HC-PCF has enabled multiple Stokes and anti-Stokes lines to be generated, spanning several octaves in frequency, using only a moderately powerful nanosecond pump source. A quantum-optical study of the Raman frequency generation process has revealed that all the components are phase-locked to one another, despite the generation being initiated by quantum noise. This makes the spectra appropriate for the generation of attosecond pulses by Fourier synthesis.

Despite the successes of gas-filled HC-PCF cells, many improvements are possible. Increasing the reflection at either end of the gas-cell, so as to enhance the cavity finesse, increase the light field intensity, and consequently strengthen the lightatom interaction, will enable new quantum-optical effects to be observed at accessible power levels. Such refinements will impact frequency standards, timing measurements, and the observation and control of ultra-fast physical phenomena.

\section{ACKNOWLEDGEMENTS}

F. Benabid is an Advanced Research Fellow of the UK Engineering and Physical Science Research Council (EPSRC). P. J. Roberts acknowledges financial support from the Danish High Technology Fund.

\section{References}

[1] F. Benabid, F. Couny, J. C. Knight, T. A. Birks, and P. S. J. Russell, "Compact, stable and efficient all-fibre gas cells using hollow-core photonic crystal fibres" Nature 434, 488-491 (2005).

[2] B. Mangan, L. Farr, A. Langford, P. J. Roberts, D. P. Williams, F. Couny, M. Lawman, M. Mason, S. Coupland, R. Flea, H. Sabert, J. C. K. T. A. Birks, and R. St. J. Philip, "Low loss ( $1.7 \mathrm{~dB} / \mathrm{km}$ ) hollow core photonic bandgap fiber" in OFC 2004 (2004).

[3] F. Benabid, J. C. Knight, G. Antonopoulos, and P. S. J. Russell, "Stimulated Raman Scattering in Hydrogen-Filled Hollow-Core Photonic Crystal Fiber" Science 298, 399-402 (2002).

[4] F. Benabid, P. S. Light, F. Couny, and P. S. J. Russell, "Electromagnetically-induced transparency grid in acetylene-filled hollow-core PCF" Opt. Express 13, 5694 (2005).

[5] F. Couny, P. S. Light, F. Benabid, and P. S. J. Russell, "Electromagnetically induced transparency and saturable absorption in all-fiber devices based on $12 \mathrm{C}_{2} \mathrm{H} 2$-filled hollow-core photonic crystal fiber" Opt. Commun 263, 28-31 (2006).

[6] S. Ghosh, J. Sharping, D. G. Ouzounov, and A. L. Gaeta, “Resonant Optical Interactions with Molecules Confined in Photonic Band-Gap Fibers" Phys. Rev. Lett. 94, 093902 (2005). 
[7] F. Couny, F. Benabid, P. J. Roberts, P. S. Light, and M. G. Raymer, "Generation and Photonic Guidance of Multi-Octave Optical-Frequency Combs" Science, 318, 1118-1121 (2007).

[8] G. S. Wiederhecker, C. M. B. Cordeiro, F. Couny, F. Benabid, S. A. Maier, J. C. Knight, C. H. B. Cruz, and H. L. Fragnito, "Field enhancement within an optical fibre with a subwavelength air core" Nat. Photon. 1, 115-118 (2007).

[9] P. Yeh and A. Yariv, "Bragg reflection waveguides" Opt. Commun. 19, 427-430 (1976).

[10] P. Yeh, A. Yariv, and E. Marom, "Theory of Bragg fiber" J. Opt. Soc. Am 68, 1196-1201 (1978).

[11] Y. Fink, J. N. Winn, S. Fan, C. Chen, J. Michel, J. D. Joannopoulos, and E. L. Thomas, "A Dielectric Omnidirectional Reflector" Science 282, 1679-1682 (1998).

[12] W. Yang, D. B. Conkey, B. Wu, D. Yin, A. R. Hawkins, and H. Schmidt, "Atomic spectroscopy on a chip" Nat. Photon. 1, 331335 (2007).

[13] T. A. Birks, P. J. Roberts, P. S. J. Russell, D. M. Atkin, and T. J. Shepherd, "Full 2-D photonic bandgaps in silica/air structures" Electron. Lett. 31, 1941-1943 (1995).

[14] G. Humbert, J. Knight, G. Bouwmans, P. Russell, D. Williams, P. Roberts, and B. Mangan, "Hollow core photonic crystal fibers for beam delivery" Opt. Express 12, 1477-1484 (2004).

[15] J. West, C. Smith, N. Borrelli, D. Allan, and K. Koch, "Surface modes in air-core photonic band-gap fibers" Opt. Express 12, 1485-1496 (2004).

[16] K. Saitoh, N. Mortensen, and M. Koshiba, "Air-core photonic bandgap fibers: the impact of surface modes" Opt. Express 12, 394-400 (2004).

[17] T. Birks, D. Bird, T. Hedley, J. Pottage, and P. Russell, "Scaling laws and vector effects in bandgap-guiding fibres" Opt. Express 12, 69-74 (2003).

[18] F. Benabid, and P. S. J. Russell, "Hollow-core PCF; progress and prospects," in Proc. SPIE, A. Adibi, S.-Y. Lin, and A. Scherer, eds. 176-190 (SPIE, San Jose, Ca, 2005).

[19] G. Antonopoulos, F. Benabid, T. A. Birks, D. M. Bird, J. C. Knight, and P. S. J. Russell, "Experimental demonstration of the frequency shift of bandgaps in photonic crystal fibers due to refractive index scaling" Opt. Express 14, 3000-3006 (2006).

[20] F. Couny, F. Benabid, P. J. Roberts, M. T. Burnett, and S. A. Maier, "Identification of Bloch-modes in hollow-core photonic crystal fiber cladding" Opt. Express 15, 325-338 (2007).

[21] P. Yeh, Optical waves in layered media (John Wiley and Sons, New York, 1988)

[22] M. A. Duguay, Y. Kukubun, T. L. Koch, and L. N. Pfeiffer, “Antiresonant reflecting optical waveguides in $\mathrm{SiO}_{2}$-Si multilayer strucutures" Appl. Phys. Lett. 49, 13-15 (1986).
[23] N. Litchinitser, S. Dunn, B. Usner, B. Eggleton, T. White, R. McPhedran, and C. D. Sterke, "Resonances in microstructured optical waveguides" Opt. Express 11, 1243-1251 (2003).

[24] N. M. Litchinister, A. K. Abeeluck, C. Headley, and B. J. Eggleton, "Antiresonant reflecting photonic crystal optical waveguides" Opt. Lett. 27, 1320-1323 (2002).

[25] T. A. Birks, G. J. Pearce, and D. M. Bird, "Approximate band structure calculation for photonic bandgap fibres" Opt. Express 14, 9483-9490 (2006).

[26] N. W. Aschcroft and N. D. Mermin, Solid state physics (Saunders College, Philadelphia, PA19105, 1976).

[27] A. W. Snyder and X. H. Zheng, "Optical fibers of arbitrary cross sections" J. Opt. Soc. Am. A 3, 600-609 (1986).

[28] A. W. Snyder and J. D. Love, Optical Waveguide Theory (Springer, 1983).

[29] T. A. Birks, J. C. Knight, and P. S. J. Russell, “Endlessly single-mode photonic crystal fiber" Opt. Lett. 22, 961-963 (1997).

[30] T. D. Hedley, D. M. Bird, F. Benabid, J. C. Knight, and P. S. J. Russell, "Modelling of a novel hollow-core photonic crystal fibre" in Quantum Electronics and Laser Science, 2003. QELS. Postconference Digest 2 (2003.)

[31] J. M. Pottage, D. M. Bird, T. D. Hedley, T. A. Birks, J. C. Knight, P. S. J. Russell, and P. J. Roberts, "Robust photonic band gaps for hollow core guidance in PCF made from high index glass" opt. Express 11, 2854-2861 (2003).

[32] J. v. Neumann and E. Wigner, Phys. Z. 30, 465 (1929).

[33] F. Capasso, C. Sirtori, J. Faist, D. L. Sivco, S. G. Chu, and A. Y. Cho, "Observation of an electronic bound state above a potential well" Nature 358, 565-567 (1992).

[34] S. John, "Strong localization of photons in certain disordered dielectric superlattices" Phys. Rev. Lett. 58, 2486-2489 (1987).

[35] E. Yablonovitch, "Inhibited Spontaneous Emission in Solid-State Physics and Electronics" Phys. Rev. Lett. 58, 2059-2062 (1987).

[36] A. Argyros and J. Pla, "Hollow-core polymer fibres with a kagome lattice: potential for transmission in the infrared" opt. Express $\mathbf{1 5}$ 7713-7719 (2007).

[37] R. Thapa, K. Knabe, M. Faheem, A. Naweed, 0. L. Weaver, and K. L. Corwin, "Saturated absorption spectroscopy of acetylene gas inside large-core photonic bandgap fiber" Opt. Lett. 31, 2489-2491 (2006).

[38] P. S. Light, F. Benabid, M. Maric, A. Luiten, and F. Couny, "Electromagnetically Induced Transparency in Rubidium-filled HC-PCF PDMS coated Hollow-Core PCF" Opt. Lett. 32, 1323 (2007).

[39] S. Ghosh, A. R. Bhagwat, C. K. Renshaw, S. Goh, A. L. Gaeta, and B. J. Kirby, "Low-Light-Level Optical Interactions with Rubidium Vapor in a Photonic Band-Gap Fiber" Phys. Rev. Lett. 97, 023603 (2006). 